\title{
What does naming a picture do? Effects of prior picture naming on recognition of identical and same-name alternatives
}

\author{
LINDA R. WARREN and JAMES W. HORN \\ University of Alabama, Birmingham, Alabama 35294
}

\begin{abstract}
Three experiments investigated the effects of naming pictures of objects during study on the subsequent recognition of physically identical, name-match, and new objects. Prior naming improved correct classification of all three item types at recognition. For line drawings and for photographs of functionally distinct objects, prior naming reduced the tendency to confuse identical and same-name alternatives. In Experiment 2, prior naming eliminated the right visual field/left hemisphere advantage for speeded recognition of name-match pictures, suggesting that prior naming reduces the likelihood that pictures are named at recognition. The implications of these results for dual-encoding (Paivio, 1971) and sensory-semantic (Nelson, Reed, \& McEvoy, 1977) models of picture and word processing are discussed. The results suggest that the semantic representations of objects that are perceptually distinct but share a common name are not identical, and that the effect of naming such objects is to insure that a distinct semantic representation becomes a part of the resulting memory code.
\end{abstract}

Since Kurtz and Hovland's (1953) original report, it has been generally acknowledged that naming a picture of an object at presentation facilitates subsequent verbal recall, as well as recognition of the picture. However, the appropriate interpretation of this effect continues to generate interest and theoretical debate. Two of the models (the dual-encoding model and the sensorysemantic model) that have been particularly successful in accounting for the current data on picture and word processing offer somewhat different explanations for the picture-naming effect.

The dual-encoding model (Paivio, 1971) assumes that perceptible qualities of objects or events are stored in an analogue imagery code, whereas names or verbal labels are stored in a verbal or linguistic code. Explicit instructions to label a picture at input are assumed to increase the likelihood that the event will be dually encoded. The resulting memory representation benefits from this code redundancy by allowing an increased probability of retrieval via the verbal code. Subsequent revisions of the dual-encoding model (Paivio, 1978; Paivio \& Desrochers, 1980), while expanding on the properties of, and relation between, the two codes, appear to retain this dual-code explanation of the picture-naming effect.

The sensory-semantic model (Nelson, Reed, \&

This research was supported in part by a faculty research grant to the author from the University of Alabama in Birmingham. The author wishes to thank Kenneth Kessler for help in data collection and Carl E. McFarland for comments on an earlier draft of this article. Reprint requests should be addressed to: Linda R. Warren, PhD, Department of Psychology, University of Alabama in Birmingham, Birmingham, Alabama 35294.
McEvoy, 1977) differs from the dual-encoding model primarily in the assumption that pictures and their verbal labels evoke distinct sensory representations, either visual or phonemic, but access a common semantic system. An additional relevant assumption of the sensorysemantic model is that a picture must first access the semantic system to determine its semantic content before the phonemic processing necessary to protuce a verbal label is initiated. Thus, instructions to label a picture at input are assumed to insure that semantic processing of the picture occurs and to increase the likelihood that semantic information becomes a part of the resulting memory code. Presumably, the sensorysemantic model would, in addition, not exclude the possibility that the additional sensory (phonemic) representation evoked by the verbal label might minimally facilitate retention.

There are conditions under which naming a picture at input does not improve subsequent recognition memory (Bahrick \& Boucher, 1968; Bartlett, Till, \& Levey, 1980). The failures to find a picture-naming effect appear to result from recognition test conditions in which old pictures must be discriminated from among visually similar alternatives that share with the target picture a common verbal label. The dual-encoding model would predict this result on the assumption that a common verbal encoding does not aid discrimination among visually similar alternatives. The sensory-semantic model would make essentially the same prediction, based on the assumption of a common semantic representation. Thus, both models and the available data are in substantial agreement that naming does not increase the discriminability among visually similar pictures that share the same verbal label. 
However, there are numerous objects in the world, and pictures of objects, that are not perceptually confusable but share the same common name. How does verbal labeling affect the memory representation of an object, or picture of an object, that shares a common name with many other perceptually distinct objects? For example, consider the assortment of shoes in a typical closet. If one of these shoes were included in a set of common objects to be studied for later recognition, it is generally assumed that labeling the selected shoe would aid in the discrimination of the shoe from a set of new objects that have different common names. The data cited above also suggest that labeling the shoe at study would not aid in discriminating the selected shoe from a perceptually similar alternative, for example, the mate to the shoe. However, would labeling the selected shoe at study aid in discriminating that shoe from other shoes in the closet, which are certainly similar to but not perceptually confusable with the studied object?

The experiments reported here deal with the effects on recognition memory of prior naming of pictures of objects that share a name with many other perceptually distinct objects. Both the dualencoding model and the sensory-semantic model assume that verbal labeling would result in an increased ability to discriminate old objects from new objects with different semantic representations and different names. However, neither model is clear as to the effect of labeling on the discrimination of old objects from new objects that share the same label. If labeling adds the same mnemonic information, regardless of the object eliciting the label, then prior naming should not aid in the discrimination among old objects and same-name alternatives. If it is assumed, according to a dual-encoding model, that the verbal code resulting from naming is the same for all objects eliciting the same name, then naming should not help in distinguishing among same-named objects. If it is assumed, according to the sensory-semantic model, that the semantic code is the same for all objects eliciting the same name, then naming should not help in distinguishing among same-named objects. Alternatively, if naming an object adds linguistic or semantic information that varies, depending upon the object eliciting the name, then prior naming should aid in the discrimination among old objects and new objects that share a common name. Experiments $1 \mathrm{a}$ and $1 \mathrm{~b}$ were designed as an explicit test of these two alternatives.

Subjects viewed line drawings (Experiment 1a) or slide photographs (Experiment $1 b$ ) of common objects. One group named the objects during presentation, and the other group did not. At recognition, subjects classified test pictures as a physical match (physically identical to a presentation object), as a name match (a physically distinct object, but one sharing the same common name as a presentation picture), or new (a new object not previously presented). Naming the pictures at input would be expected to aid in the classifi- cation of new pictures, according to both dual-encoding and sensory-semantic models. Of particular interest was the effect of naming on the classification of physicalmatch and name-match pictures.

\section{EXPERIMENT 1A}

\section{Method}

Design. During exposure of the study pictures, subjects were instructed to study and name each picture (name) or to study each picture (no name). Recognition was tested, either immediately or after a 24 -h delay, on a list composed of study list pictures (physical match), different pictures with the same name (name match), and pictures of different objects (new). This resulted in a 2 by 2 by 3 factorial design, with orientation task (name vs. no name) and test time (immediate vs. delayed) as between-subjects factors and test item type (physical match, name match, or new) as a within-subjects factor.

Subjects. Forty-eight student volunteers, who served in the experiment as part of a course requirement, were randomly assigned to the four treatment conditions.

Materials. There were six study lists, each composed of 30 line drawings of common objects, and two test lists, each composed of three sets of 15 line drawings. There were three semirandom orders of each test list, arranged such that items from each of the three sets occurred equally often in each third of the test list. Study and test lists were paired such that each of the three test list sets was used equally of ten as physical matches, name matches, and new items across subjects. The materials used in Experiment 1a are presented in Figure 1.

Procedure. Subjects were seated in front of a rear-projection screen. Slides of the 30 -item study set were presented via a Kodak Carousel projector at a 2-sec rate. Subjects were instructed to study each picture and, in the name condition, to call out the common name of each object as it was presented. The names were recorded and compared with the experimenter's name list to check for any misidentifications. Naming was not mentioned in the no-name condition. Following study list presentation, subjects were told to relax for a few minutes (immediate test) or were dismissed and told to return at the same time the next day (24-h test). After $4 \mathrm{~min}$ in the immediate group or upon return in the delayed group, recognition test instructions were read. Subjects were told they would see a set of pictures, some of which would be identical to pictures on the study list, some of which would be different pictures that would have the same name as pictures on the study list, and some of which would be pictures of new objects. Subjects were assured that name matches would look noticeably different from the study list items, so that if they thought they recognized a picture but were unsure about tiny visual details, they should call it "old." Subjects were given an answer sheet with 45 spaces and columns labeled "Old," "Same Name," and "New." They were told to call out the name of each picture as it was presented and to check the appropriate column for each picture. The test list pictures were presented in the same way as study pictures, but at a 4-sec rate. Again, the subjects' names for each picture were recorded for comparison with study list names.

\section{Results}

There were only 20 naming errors (differences in experimenter and subject names for pictures) across conditions, items, and subjects, 4 errors during the study list and 16 on the test list. Only four of these errors resulted in a test list classification error, and these items (name matches called new) were scored as correct new classifications. 


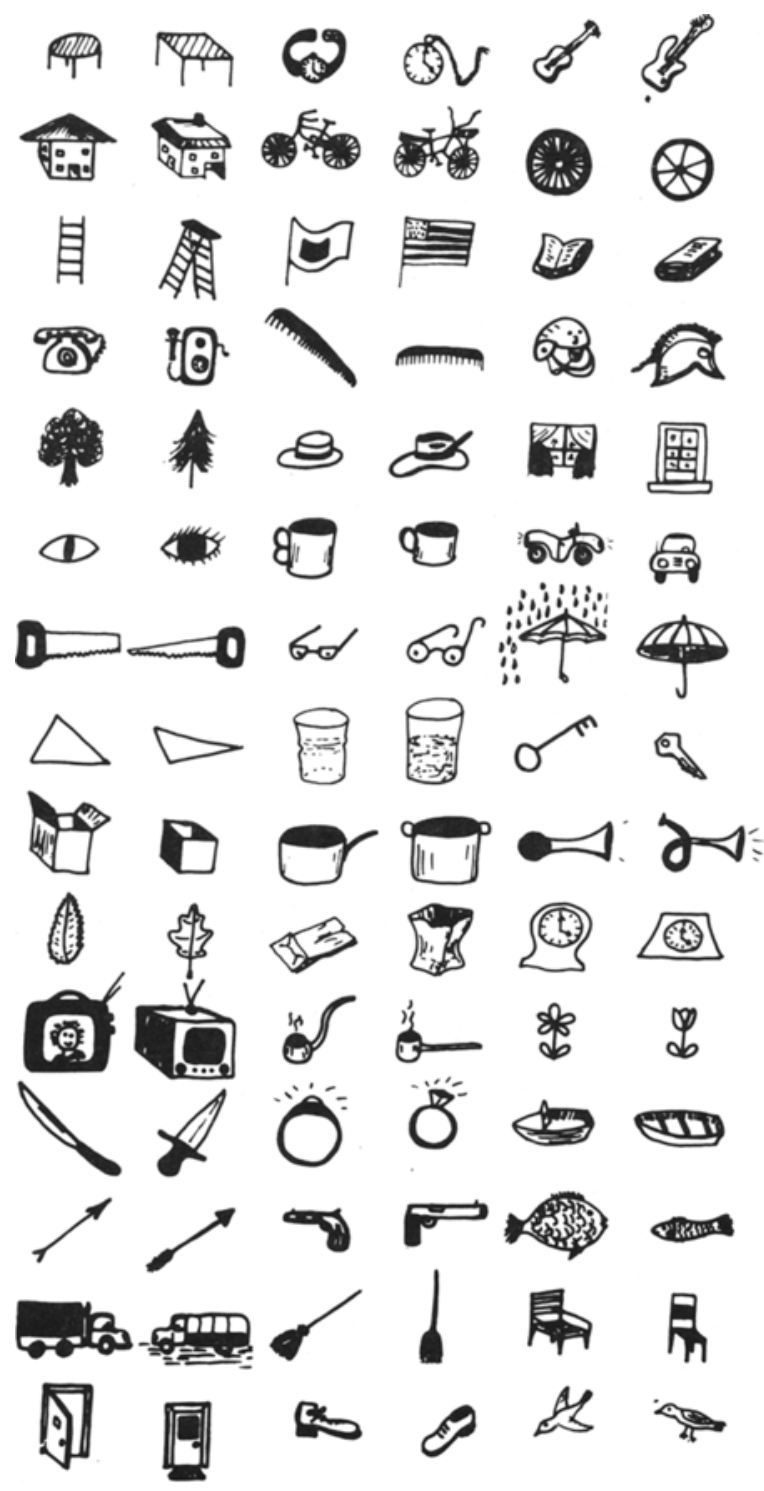

Figure 1. Same-named alternatives used as name-match, physical-match, or new items in Experiment 1a.

Mean classification responses for each item type at recognition are presented in Table 1 . A 2 by 2 by 3 ANOVA on correct responses, with name/no name, time of test, and item type (physical match, name match, new) as the three factors, resulted in significant main effects for all three factors. Naming the pictures during study increased correct recognition $[F(1,44)=$ 13.34, $\mathrm{MSe}=3.50, \mathrm{p}<.01]$, immediate recognition was better than delayed recognition $[F(1,4 \xi)=17.53$, $\mathrm{MSe}=3.50, \mathrm{p}<.01]$, and classification of name matches was poorer than classification of physical matches or new pictures $[\mathrm{F}(2,88)=28.18$, MSe $=3.91, \mathrm{p}<.01]$. The Name/No Name by Time of Test interaction was also significant $[\mathrm{F}(1,44)=5.36, \mathrm{MSe}=3.50, \mathrm{p}<.05]$, indicating a larger difference between the naming conditions after $24 \mathrm{~h}$. However, this could be a result of ceiling effects on the immediate test. No other interactions were significant. The absence of a Name/No Name by Item Type interaction indicates that the beneficial effects of naming improved correct classification of physical and name matches at least as much as it did that of new items.

As Table 1 shows, there were relatively few errors to physically identical and new pictures at either retention interval. For no-name conditions, the most classification errors for the name-match pictures resulted from an incorrect classification response of "new" (means of 1.42 physical-match responses vs. 2.42 new responses) at the immediate test, and from an incorrect classification response of "physical match" after a 24-h delay (means of 4.08 physical-match responses vs. 2.67 new responses). How are these recognition failures affected by verbal labeling of the original picture set at presentation? On the immediate test, naming served to reduce the tendency to classify name-match pictures as "new." This is exactly the result predicted by both the dualencoding and the sensory-semantic models. However, after the 24-h delay, naming pictures at input reduced both the tendency to classify name matches as "new" and the tendency to classify name matches as "physical match" or "old." The most reasonable interpretation of the latter effect is that naming pictures at input increases discriminability among old and same-name alternatives on either the imagery or sensory-semantic codes. There is, however, in retrospect, a problem with the design of Experiment 1a that limits somewhat the strength of this conclusion. In Experiment 1a, all subjects were required

Table 1

Experiment 1a: Mean Numbers of Classification Responses (Correct Responses and Errors) to Three Types of Line Drawings During Recognition as a Function of Presentation Condition (No Name, Name) and Time of Retention Test (Immediate, 24-h Delay)

\begin{tabular}{lrcr}
\hline & \multicolumn{3}{c}{ Classification Response } \\
\cline { 2 - 4 } Item Type & $\begin{array}{c}\text { Physical } \\
\text { Match }\end{array}$ & $\begin{array}{c}\text { Name } \\
\text { Match }\end{array}$ & New \\
\hline Physical Match & 13.50 & 1.08 & .42 \\
Name Match & 1.42 & 11.17 & 2.42 \\
New & .00 & 1.50 & 13.50 \\
& \multicolumn{3}{c}{ Immediate/No Name } \\
Physical Match & 13.83 & .75 & .42 \\
Name Match & 1.67 & 12.33 & 1.00 \\
New & .00 & 1.75 & 13.25 \\
& \multicolumn{4}{c}{ Delay/No Name } \\
Physical Match & 12.08 & 1.58 & 1.33 \\
Name Match & 4.08 & 8.25 & 2.67 \\
New & 1.50 & 1.75 & 11.75 \\
& \multicolumn{3}{c}{ Delay/Name } \\
Physical Match & 13.92 & .75 & .33 \\
Name Match & 2.67 & 10.42 & 1.92 \\
New & .58 & 1.08 & 13.33 \\
\hline
\end{tabular}


to label the pictures at test, but only the subjects in the name conditions labeled pictures at study. It is possible that the introduction of the naming requirement at test disrupted the recognition performance of the no-name, more than the name, subjects. The purpose of the naming requirement at test was only to insure that both groups were using the same common name for identical and same-name alternatives. Essentially, the same information can be inferred from the study list names, since items serving as identical and same-name alternatives were counterbalanced across subjects. Thus, in Experiment $1 \mathrm{~b}$, subjects were not asked to name the items at test.

It is also apparent, from inspection of Figure 1, that the item pairs used as materials in Experiment 1a differed widely in their visual and "semantic" similarity. If prior labeling of an object affects the discriminability of the sensory or semantic mnemonic codes, then the more objects differ in these dimensions, the more discriminable they should become as a function of prior naming. Thus, in Experiment $1 \mathrm{~b}$, the similarity of same-name alternatives was included as a variable. Because it proved difficult to incorporate enough detail to vary semantic similarity within a class of same-named objects using line drawings but was relatively easy using real objects, materials for Experiment $1 \mathrm{~b}$ consisted of slide photographs.

\section{EXPERIMENT 1B}

\section{Method}

Design. During exposure of the study slides, subjects were instructed to study and name each slide (name) or to study each slide (no name). Recognition was tested, after a 48-h delay, on a list composed of slides of study list objects (object match), slides of different objects with the same common name (name match), and slides of differently named objects (new). Namematch items were either functionally quite similar (same subcategory) or functionally somewhat different (different subcategory) from study list objects. This resulted in a 2 by 2 by 3 factorial design with orientation task (name vs. no name) and similarity of name-match items (same or different subcategory) as between-group factors and test item type (object match, name match, new) as a within-group factor.

Subjects. Sixty student volunteers, serving in the experiment as part of a course requirement, were randomly assigned to the four treatment conditions.

Materials. There were six study lists, each composed of 24 slide photographs of common objects, and a single test list composed of a partially random order of three sets of 12 slides. Three of the study lists served as materials for the samesubcategory groups, and the other three served as materials for the different-subcategory groups. The test list consisted of slightly different photographs of study list objects (object match), photographs of similar or different objects that shared the same common name as study list objects (name match), and photographs of new objects with different common names (new). Object type for each test list slide was defined in terms of the prior study list. Study lists were constructed such that each of the three test list subsets was used equally of ten as object-match, name-match, and new items across subjects. A list of the common names of the objects photographed and the relation between name-match objects is presented in Table 2 . Objects were pretested to insure that items in both subcategories elicited the same common name. Some of the object groups elicited more than one common name. However, pretesting indicated that, when asked to name the objects, a given individual used the same name for members of both subcategories. Items were selected such that objects within a subcategory differed primarily in appearance (color and either shape or design), whereas between subcategories, objects differed in both appearance and specialized function.

Procedure. General instructions to subjects and procedures were identical to those in Experiment 1a, with the following exceptions. Slides were presented at a 5 -sec rate at both study and test. Recognition was tested after a 48-h delay, and subjects did not name the slides during the recognition test.

\section{Results}

Eight of the object groups elicited more than one common name, as indicated in Table 2. However, the distributions of responses to the items within each object group were virtually identical; that is, a particular common name was equally likely to be elicited by items of either subcategory. Because pretesting had indicated that a given individual was likely to be consistent in the use of a particular common name, no attempt was made to treat these items differently in the subsequent analyses.

Mean classification responses for each item type at recognition are presented in Table 3 . A 2 by 2 by 3 ANOVA was performed on correct responses, with name/no name and same vs. different name-match items as between-subjects factors and item type (object match, name match, new) as a within-subjects factor nested under the same-different factor.

Naming the objects during study increased correct recognition $[F(1,56)=11.28, \quad \mathrm{MSe}=4.17, \mathrm{p}<.01]$. The type of subcategory relation between study list and name-match items did not affect the overall number of correct classification responses to the three item types $[F(1,56)<1]$, and the interaction between naming and the same/different-subcategory relation was not significant $[F(1,56)<1]$. Classification of name-match items was poorer than classification of object-match or new pictures for both same-subcategory groups $[F(2,56)=36.85, \mathrm{MSe}=3.00, \mathrm{p}<.01]$ and different-subcategory groups $[\mathrm{F}(2,56)=25.77, \mathrm{MSe}=$ $4.45, p<.01]$, and naming did not interact with item type for either the same- or different-subcategory groups.

Although the statistical analysis of correct responses supports the interpretation that naming study list objects improves the classification of similar same-name alternatives as much as it does different same-name alternatives, inspection of the error patterns in Table 3 suggests that the benefits of naming occur for different reasons. When name-match items came from the same subcategory as study list items, the effect of prior naming was to reduce the confusion between new objects and the other two types. Prior naming did not appear to aid in the discrimination between object-match and similar name-match alternatives. However, when name-match items came from a subcategory different from that of study list items, prior naming appeared to reduce confusion between object-match and name-match items, 
Table 2

Common Names and Types of Objects in the Two Different Subcategories Used as Name-Match Items in Experiment 1b

\begin{tabular}{|c|c|c|}
\hline Object Group (Common Name) & \multicolumn{2}{|c|}{ Types (Subcategories) } \\
\hline clock & alarm & wall \\
\hline coathanger/hanger/clotheshanger & wire & wooden \\
\hline doll & baby & rag \\
\hline shovel & sandbox & garden \\
\hline cap/hat & baseball & knit \\
\hline boot & lady's & hiking \\
\hline hammer & tack & claw \\
\hline spoon & wooden & plastic \\
\hline teapot & copper & ceramic \\
\hline screwdriver & regular & pocket \\
\hline fork & cooking & table \\
\hline keys & assorted & car \\
\hline scissors & sewing & nail \\
\hline ashtray & decorative & glass \\
\hline lightbulb/bulb & nightlight & lamp \\
\hline knife & table & bread \\
\hline bucket & child's & scrub \\
\hline umbrella & regular & folding \\
\hline rake & leaf & garden \\
\hline paintbrush & artist's & wall \\
\hline life-vest/life-jacket & coastguard & ski \\
\hline battery & flashlight & lantern \\
\hline saw & hand & narrow blade \\
\hline cup & tea & paper \\
\hline book & hardback & child's \\
\hline tape & masking & cellophane \\
\hline purse/handbag/pocketbook & shoulder strap & clutch \\
\hline eyeglasses/glasses & wire rim & plastic frame \\
\hline shirt & dress & knit \\
\hline wire & insulated & strand \\
\hline pliers & needlenose & regular \\
\hline watch & man's & lady's \\
\hline calculator & handheld & desk \\
\hline trashcan/wastebasket/garbage can & round & swingtop \\
\hline comb & pocket & handle \\
\hline pen/inkpen & ballpoint & fountain \\
\hline
\end{tabular}

as well as between new items and the other two types.

Thus, the error data of both Experiments $1 \mathrm{a}$ and $1 \mathrm{~b}$ suggest that prior naming of pictured objects improves subsequent discrimination between pictures of old objects and same-name alternatives. However, the effect appears to be limited to same-name items that differ somewhat in function, as well as appearance.

A possible interpretation of the error data, within the context of the sensory-semantic model, is that a picture rarely results in the identical semantic representation as a single word and that pictures of two visually and functionally distinct objects with the same name do not result in identical semantic representations. This possibility will be explored in more detail in the Discussion section. For now, it is enough to assume that visually distinct instances of a concept that share a common name are members of semantic subcategories and that the semantic representation of an instance preserves to some extent information about subcategory membership. Thus, two instances of a concept sharing a common name would have similar, but frequently distinct, semantic representations. If naming a picture strengthens its own distinct semantic representation, as well as the more general semantic representation that would correspond to the picture's name, then physicalmatch pictures could be discriminated from namematch pictures on the basis of semantic as well as sensory information.

An important implication of this alternative assumption is that correct classification of the three picture types at test could result from an analysis of visual configuration plus minimal semantic processing, without the necessity of actually accessing the phonemic information corresponding to the pictures' names. According to this proposed modification of the sensorysemantic model, classification based only on visual configuration and early semantic processing would be expected to be more successful in the name conditions than in the no-name conditions, because of the increased semantic processing at study, thus reducing the likelihood that the phonemic representation would be accessed in the name conditions. This assumption is, of course, inconsistent with the Paivio (1971) dualencoding model, which assumes that the verbal code 
Table 3

Experiment 1b: Mean Numbers of Classification Responses (Correct Responses and Errors) to Three Types of Photographs During Recognition as a Function of Presentation Condition (No Name, Name) and Relation of Name-Match Items to Study List Items (Same Subcategory, Different Subcategory)

\begin{tabular}{lccr}
\hline & \multicolumn{3}{c}{ Classification Response } \\
\cline { 2 - 4 } Item Type & $\begin{array}{l}\text { Object } \\
\text { Match }\end{array}$ & $\begin{array}{c}\text { Name } \\
\text { Match }\end{array}$ & New \\
\hline & & No Name & \\
Object Match & 8.67 & 2.40 & .93 \\
Name Match* & 4.40 & 5.80 & 1.80 \\
New & 1.33 & 2.00 & 8.67 \\
& & Name & \\
Object Match & 9.27 & 2.47 & .27 \\
Name Match* & 4.60 & 6.00 & 1.40 \\
New & .67 & 1.13 & 10.20 \\
& & No Name & \\
Object Match & 8.40 & 2.40 & 1.20 \\
Name Match $\dagger$ & 2.80 & 5.13 & 4.07 \\
New & 1.53 & 1.13 & 9.33 \\
& & Name & \\
Object Match & 10.20 & 1.53 & .27 \\
Name Match $\dagger$ & 2.53 & 6.87 & 2.60 \\
New & 1.00 & 1.40 & 9.60 \\
\hline
\end{tabular}

*Same subcategory. TDifferent subcategory.

must be accessed at test for any beneficial effects of prior naming to occur.

Experiment 2 was designed as a test of the hypothesis that naming the pictures at study reduces the likelihood that pictures are named during a speeded recognition test. Subjects viewed line drawings of common objects under presentation conditions identical to those in Experiment 1a. One group named the pictures during presentation, and one group did not. At recognition, probe pictures (physical match, name match, or new) were presented laterally, either to the left or to the right visual field, and subjects classified the pictures with a buttonpress response. Our reasoning was that if subjects were naming the pictures at recognition, response times should be shorter for pictures presented to the right visual field (left hemisphere) than for those presented to the left visual field (right hemisphere), under the assumption that language is normally lateralized in the left hemisphere of right-handed people. However, if subjects were not naming the pictures at recognition, either no effect of visual field or the typical left visual field/right hemisphere advantage for pictorial-spatial material should occur (Geffen, Bradshaw, \& Wallace, 1971).

\section{EXPERIMENT 2}

\section{Method}

Design. During exposure of the study pictures, subjects were again instructed to study and name aloud each picture (name) or only to study each picture (no name). Recognition was tested immediately on a list composed of physical-match, name-match, and new pictures, presented equally often to the left and right visual fields. This resulted in a 2 by 3 by 2 factorial design, with orientation task (name or no name) as a between-subjects factor and picture type and visual field as within-subjects factors.

Subjects. Forty-two righthanded student volunteers, serving in the experiment as part of a course requirement, were randomly assigned to the two orientation task conditions.

Materials. There were three study lists, each composed of 24 line drawings of common objects, and a single test list composed of three sets of 12 line drawings. The drawings consisted of those items least likely to result in naming errors from Experiment 1a. Line drawings were used, rather than slide photographs, because they allowed easier adjustment of the visual angle for control of the visual field manipulation. The test list was presented in three semirandom orders, arranged such that physical matches from the study list and items from each test set were distributed equally throughout the test list and were presented equally of ten to the right and left visual fields. As in Experiments $1 \mathrm{a}$ and $1 \mathrm{~b}$, study lists were paired with the test list such that, across subjects, each picture was used equally of ten as a physical-match, name-match, and new item in both the left and right visual fields.

Procedure. Subjects sat in a comfortable chair, with chin resting in a chin rest mounted $24 \mathrm{in}$. in front of a rear-projection viewing screen. The first three fingers of each hand rested on response buttons arranged in a slight semicircle. The three response buttons for each hand were wired to separate signal lights and a single Hunter millisecond timer for each hand. Thus, depression of any button was signaled by one of six lights and stopped the right- or left-hand timer. Before beginning the study phase of the picture task, subjects were given practice on the use of the response apparatus. Each subject was assigned the labels "old," "same," and "new" for the two first-, second-, and third-finger buttons. The labels for each pair of fingers were counterbalanced across subjects. During practice, the capital letters $\mathrm{O}, \mathrm{S}$, and $\mathrm{N}$ were presented $5 \mathrm{deg}$ to the left or right of a central fixation point, and the subjects' task was to press simultaneously the two first-, second-, or third-finger buttons corresponding to the appropriate label, as rapidly as possible. Letters were presented for $200 \mathrm{msec}$ duration at a $10-\mathrm{sec}$ rate via a Kodak Carousel projector and Gerbrands tachistoscopic shutter. Subjects practiced on this letter-response task until performance was virtually error free and coordination of response to the corresponding buttons of the two hands was virtually simultaneous.

Following the practice task, the study phase of the recognition task was presented. Pictures were presented, in the center of the screen, for $6 \mathrm{sec}$ each. Subjects were instructed to study and try to remember each picture (no name) or to call out the name of each picture, study it, and try to remember it (name).

Following the study task, subjects were instructed about the nature of the recognition task, the correspondence of the buttons to picture type (i.e., old-a physical match; same-physically different, but same name as a study picture; and new -a different picture and name), and the importance of keeping their eyes fixated on the central dot and of pressing the appropriate response keys rapidly and simultaneously. Pictures were presented to the left or right visual field (centered $5 \mathrm{deg}$ to the left or right of fixation, covering no more than $5 \mathrm{deg}$ of visual angle) at $200 \mathrm{msec}$ duration and a 6 sec rate.

\section{Results}

In Experiment 2, there were no study list naming errors (differences in subject- and experimenter-assigned names for pictures) in the name condition.

Mean classification responses for each item type during recognition are presented in Table 4 . A 2 by 2 by 3 ANOVA on correct responses, with name/no name, 
field of presentation, and item type (physical match, name match, new) as the three factors resulted in significant main effects for naming $[F(1,40)=13.06, \mathrm{MSe}=$ $.92, \mathrm{p}<.01]$ and item type $[\mathrm{F}(2,80)=19.85, \mathrm{MSe}=.74$, $p<.01]$. The main effect of field of presentation and effects of all interactions did not approach significance.

The analysis of response times for correct classification responses was based on each subject's median response time to items of each type, in each visual field. Means of median response times are presented in Table 5 . A 2 by 2 by 3 by 2 ANOVA was performed on median response times in seconds, with name/no name, field of presentation, item type, and response hand (subjects attempted to respond simultaneously with both hands) as the four factors. The main effects of naming $[\mathrm{F}(1,40)=4.95, \mathrm{MSe}=1.58, \mathrm{p}<.05]$ and item type $[F(2,80)=19.39, \mathrm{MSe}=.18, \mathrm{p}<.01]$ were again significant. The main effect of visual field was not significant. The effect of response hand, a $10-\mathrm{msec}$ advantage for the right hand over the left, was significant $[F(1,40)=5.89, \mathrm{MSe}=.0007, \mathrm{p}<.05]$, but this effect did not interact with any other variables. The only significant interaction was the interaction of Naming by Item Type by Field of Presentation $[\mathrm{F}(2,80)=3.88$, $\mathrm{MSe}=.0004, \mathrm{p}<.051$.

Table 4

Experiment 2: Mean Numbers of Classification Responses (Correct Responses and Errors) to Three Types of Items During Recognition as a Function of Condition (No Name, Name) and Visual Field of Presentation

\begin{tabular}{|c|c|c|c|c|c|c|}
\hline \multirow[b]{3}{*}{ Item Type } & \multicolumn{6}{|c|}{ Classification Response } \\
\hline & \multicolumn{2}{|c|}{ Physical Match } & \multicolumn{2}{|c|}{ Name Match } & \multicolumn{2}{|c|}{ New } \\
\hline & $\mathrm{LF}$ & RF & LF & RF & LF & RF \\
\hline & \multicolumn{6}{|c|}{ No Name } \\
\hline Physical Match & 4.14 & 4.19 & 1.48 & 1.43 & .38 & .38 \\
\hline Name Match & 1.05 & 1.43 & 3.90 & 3.52 & 1.05 & 1.05 \\
\hline \multirow[t]{2}{*}{ New } & .19 & .24 & .71 & .80 & 5.10 & 4.95 \\
\hline & \multicolumn{6}{|c|}{ Name } \\
\hline Physical Match & 4.86 & 4.67 & .95 & 1.00 & .19 & .33 \\
\hline Name Match & .76 & .90 & 4.43 & 4.62 & .81 & .48 \\
\hline New & .10 & .14 & .24 & .52 & 5.67 & 5.33 \\
\hline
\end{tabular}

Note $-L F=$ left visual field $; R F=$ right visual field.

Table 5

Experiment 2: Mean of Median Response Times (for Correct Responses) in Milliseconds to Three Item Types as a Function of Condition (No Name, Name) and Visual Field of Presentation

\begin{tabular}{lccccc}
\hline & \multicolumn{2}{c}{ No Name } & & \multicolumn{2}{c}{ Name } \\
\cline { 2 - 3 } \cline { 5 - 6 } Item Type & LF & RF & & LF & RF \\
\hline Physical Match & 1505 & 1562 & & 1272 & 1328 \\
Name Match & 1895 & 1759 & & 1567 & 1571 \\
New & 1585 & 1664 & & 1357 & 1385 \\
Mean & 1662 & 1662 & & 1399 & 1428 \\
\hline
\end{tabular}

Note- $L F=$ left visual field $R F=$ right visual field.
An analysis of simple interaction effects of Item Type by Field of Presentation for name and no-name conditions resulted in an Item Type by Field interaction only for the no-name condition $[\mathrm{F}(2,40)=3.42$, $\mathrm{MSe}=$ $.09, \mathrm{p}<.05]$. The significant interaction reflects the fact that only the name-match items in the no-name condition resulted in a right visual field/left hemisphere advantage. All other items were classified as quickly when presented to the left visual field as when presented to the right visual field. This result is consistent with the assumption that old and new items in the no-name condition and all items in the name conditions were typically classified on the basis of their sensory and semantic representations without the necessity of accessing their respective verbal labels. Apparently, only the name-match items in the no-name condition were named at test. Presumably, these items are named because neither the stored visual information nor semantic information is sufficient for the classification decision. The relatively longer classification times for these items indicate the difficulty of the decision.

\section{DISCUSSION}

The major finding of the experiments reported here is that naming a picture of an object during presentation aids in the classification of test list pictures as a physicalmatch, name-match, or new item. While both the dualencoding model (Paivio, 1971) and the sensory-semantic model (Nelson et al., 1977) would predict that naming would aid in the discrimination of new pictures from physical-match and name-match pictures, it is not clear that either model can account for beneficial effects of naming on the discrimination between physical. match and name-match items. The dual-encoding model assumes that the beneficial effects of prior naming are due to the availability of the name as a recognition cue, which should not help in distinguishing physicalmatch and name-match items. The sensory-semantic model assumes that the beneficial effects of prior naming are due to increased availability of the semantic code. However, this code, at least for simple line drawings, is assumed to be identical for physical-match and name-match items and should not aid in distinguishing between them.

A second finding of interest is that prior naming eliminates the right visual field/left hemisphere advantage for response time to name-match pictures, suggesting, somewhat paradoxically, that naming pictures at input reduces the likelihood that pictures are named during a speeded recognition test. This result is consistent with the assumption of the sensory-semantic model that naming pictures at input insures that semantic processing of the picture occurs. Presumably, then, at test, correct classification of name-match items can occur on the basis of stored sensory and semantic information about presentation pictures. However, for facilitation 
of discrimination between physical-match and namematch items to occur as a function of naming presentation pictures, it must be assumed that naming results in a more discriminable representation at either the sensory or the semantic level.

These two possible effects of labeling have been referred to in the shape recognition literature as the discriminating function and the categorizing function of labels, respectively (Nagae, 1980). The discriminating function of a label serves to direct the subject's attention to distinctive features of the stimulus and, thus, to enhance the resulting sensory representation. Associating labels with shape stimuli does result in superior recognition memory for distinctive features of shapes in children (Nagae, 1976). The categorizing function of a label has been assumed to allow classification of shapes on the basis of distinctive features suggested by the label. Evidence for the categorizing function of labels comes from experiments showing that recognition errors reflect the use of distinctive features suggested by the label as the basis for classification (Daniel, 1972).

This description of the categorizing function of labels is consistent with the assumption of the sensorysemantic model that items sharing the same verbal label should share the same semantic classification. It seems obvious that a principal function of a verbal code is the common classification by name of conceptually equivalent but perceptually distinct objects or events. What is at issue is whether information that distinguishes among objects that share a common verbal label is also a part of the semantic code, and whether naming an object increases the likelihood that such information becomes a part of the resulting semantic representation of that object. In Rosch's (1978) terminology, labels for objects appear to be labels for categories that function at the "basic level of abstraction," that is, categories that carry the most information, are the most differentiated from each other, and for which a concrete image of the category as a whole can be formed. However, basic-level categories were composed of subordinate categories, categories that preserve information about the distinctiveness of objects that share a common name. For example, a schematic drawing of a lady's shoe and a man's shoe both typically evoke the common verbal label "shoe." The question is under what conditions does the presentation of a drawing of a lady's shoe evoke not only semantic information about shoes in general, but also about ladies' shoes in particular? Nelson et al. (1977, pp. 486-494) acknowledge that "the meaning representation for a picture may be more differentiating and effective than that associated with its label," but they then go on to claim that "a primary assumption of the model is that functional semantic codes for simple pictures and their corresponding verbal labels are identical." The results of this study on picture naming, and of other recent studies comparing the processing of pictures vs. words (Durso \& Johnson, 1979; Guenther \& Klatzky, 1977), support the first statement but appear to be inconsistent with the second. Guenther and Klatzky assume that picture classification ordinarily makes greater use of information about physical properties of the object represented than does word classification. Durso and Johnson propose that pictures are something like words in context. Pictures generate semantic representations that can be characterized by some, but not all, of the components of the concept it represents. Our interpretation of the picture-naming effect is consistent with these interpretations of picture processing. We assume that semantic processing of pictures typically involves activation of information at the "basic level of abstraction," but also information about subcategory membership. The consequence of naming a picture at presentation is to insure that such activation occurs and becomes a part of the resulting memory representation.

An alternative interpretation of the picture-naming effect that, however, cannot be eliminated on the basis of these experiments, is that information about the cognitive operations performed at input (naming) is preserved in memory (Durso \& Johnson, 1980) and serves in the name conditions as a cue for discriminating physical-match from name-match pictures. There is accumulating evidence not only that such information is available in memory, but also that the act of generating such information may increase the availability of the resulting memory representation (McFarland, Frey, \& Rhodes, 1980; Slamecka \& Graf, 1978). Thus, the beneficial effects of naming a picture, for subsequent recognition, may be due in part to the recollection that such an act (naming) did or did not occur in response to a particular picture.

\section{REFERENCES}

Bahrick, H. P., \& Boucher, B. Retention of visual and verbal codes of the same stimuli. Journal of Experimental Psychology, $1968,78,417-422$.

BartletT, J. C., Till, R. E., \& Levy, J. C. Retrieval characteristics of complex pictures: Effects of verbal encoding. Journal of Verbal Learning and Verbal Behavior, 1980, 19, 430-449.

DANIEL, T. C. Nature of the effect of verbal labels on recognition memory for form. Journal of Experimental Psychology, 1972, 96, 152-157.

Durso, F. T., \& Johnson, M. K. Facilitation in naming and categorizing repeated pictures and words. Journal of Experimental Psychology: Human Learning and Memory, 1979, 5, 449-459.

Durso, F. T., \& Johnson, M. K. The effects of orienting task on recognition, recall, and modality confusion of pictures and words. Journal of Verbal Learning and Verbal Behavior, $1980,19,416-429$.

Geffen, G., Bradshaw, J. L., \& Wallace, G. Interhemispheric effects on reaction time to verbal and nonverbal visual stimuli. Journal of Experimental Psychology, 1971, 87, 415-422.

Guenther, R. K., \& KLATZKY, R. L. Semantic classification of pictures and words. Journal of Experimental Psychology: Human Learning and Memory, 1977, 3, 498-514.

Kurtz, K. H., \& Hovland, C. I. The effect of verbalization during observation of stimulus objects upon accuracy of recog- 
nition and recall. Journal of Experimental Psychology, 1953, 45, 157-164.

McFarland, C. E., Frey, T. J., \& Rhodes, D. P. Retrieval of internally versus externally generated words in episodic memory. Journal of Verbal Learning and Verbal Behavior, $1980,19,210-225$.

NAGAE, S. Effects of labeling on shape recognition in children. Japanese Journal of Psychology, 1976, 47, 92-96.

NAGaE, S. Nature of discriminating and categorizing functions of verbal labels on recognition memory for shape. Journal of Experimental Psychology: Human Learning and Memory, $1980,6,421-429$.

Nelson, D. L., Reed, V. S., \& McEvoy, C. L. Learning to order pictures and words: A model of sensory and semantic coding. Journal of Experimental Psychology: Human Learning and Memory, 1977, 3, 485-497.
Paivio, A. Imagery and verbal processes. New York: Holt, Rinehart \& Winston, 1971.

Paivio, A. Mental comparisons involving abstract attributes. Memory \& Cognition, 1978, 6, 199-208.

Paivio, A., \& Deshochers, A. A dual-coding approach to bilingual memory. Canadian Journal of Psychology, 1980, 34, 388-399.

Rosch, E. Principles of categorization. In E. Rosch \& B. B. Lloyd (Eds.), Cognition and categorization. Hillsdale, N.J: Erlbaum, 1978.

Slamecka, N. J., \& Graf, P. The generation effect: Delineation of a phenomenon. Journal of Experimental Psychology: Human Learning and Memory, 1978, 4, 592-604.

(Received for publication February 5, 1981; revision accepted December 22, 1981.) 